\title{
Cambio y continuidad en las políticas de ciencia: diseño de los instrumentos de financiación de la investigación universitaria en Galicia (2005-2018)
} Change and continuity in science policy: The design of funding instruments for university research in Galicia (2005-2018)

\author{
MANUEL PEREIRA-PUGA \\ Instituto de Políticas y Bienes Públicos. CSIC \\ LUIS SANZ-MENÉNDEZ \\ Instituto de Políticas y Bienes Públicos. CSIC
}

\section{Cómo citar/Citation}

Pereira-Puga, M. y Sanz-Menéndez, L. (2020). Cambio y continuidad en las políticas de ciencia: Diseño de los instrumentos de financiación de la investigación universitaria en Galicia (2005-2018). Revista Española de Ciencia Política, 54, $151-171$. Doi: https://doi.org/10.21308/recp.54.06

\section{Resumen}

El objetivo de esta nota de investigación es estudiar el diseño y evolución de los instrumentos de financiación de la investigación universitaria de un Gobierno autonómico (Xunta de Galicia) durante el periodo 2005-2018. Se presentan los principales factores y procesos asociados al diseño de las políticas científicas, desde una perspectiva histórica. Para ello se han analizado múltiples fuentes documentales y se han realizado entrevistas semiestructuradas, así como reuniones con actores relacionados con la cartera de instrumentos. Los principales resultados ponen de manifiesto que: a) las decisiones tomadas sobre el diseño e implementación de instrumentos se ven condicionadas por interacciones continuas entre actores; b) la procedencia de los responsables políticos tiene un impacto en la configuración de las actividades de investigación; c) los entornos institucionales y organizativos generan oportunidades y, al tiempo, constriñen la acción de los actores políticos, y d) las conductas de reducción de costes administrativos y de gestión promueven el diseño y adopción de instrumentos con múltiples objetivos. Además, se constatan las consecuencias estructurantes de las coyunturas críticas en la continuidad de las políticas y en los diseños institucionales y organizativos, así como la existencia de procesos de sustitución y acumulación de instrumentos.

Palabras clave: cambio en las políticas, coyunturas críticas, dependencia de la senda, organización de los Gobiernos, instrumentos de política pública, políticas de $\mathrm{I}+\mathrm{D}$, investigación universitaria, Galicia. 


\begin{abstract}
The objective of this research note is to study the design and evolution of the instruments for university research-funding by a regional government (Xunta de Galicia) during the period 2005-18. It shows the main factors and processes associated to the design of science policies from a historical perspective. For this purpose, multiple documentary sources have been analyzed and semi-structured interviews as well as meetings with actors involved in the instrument portfolio have also been carried out. The key findings show that: a) decisions about the design and implementation of instruments are influenced by continuous interactions among actors; b) the policy makers' origins have an impact on the set up of research activities; c) institutional and organizational environments generate opportunities but, at the same time, they constrain the action of political actors; and d) behaviors oriented to reduce administrative and management costs promote the design and implementation of instruments with multiple objectives. Furthermore, it can be confirmed the structuring consequences of critical junctures on the continuity of policies and institutional and organizational designs, as well as the existence of processes of substitution and accumulation of instruments.
\end{abstract}

Keywords: policy change, critical junctures, path dependency, government organization set up, public policy instruments, R\&D policy, university research, Galicia.

\title{
INTRODUCCIÓN
}

Desde hace años, en muchos países, los Gobiernos han intentado dirigir e influir en las actividades de investigación de las universidades y han llevado a cabo reformas para promover modelos más gerenciales (Braun y Merrien, 1999) o para favorecer su conducta estratégica (Whitley, 2011).Tales cambios han otorgado un mayor peso a la evaluación y a la medición de resultados (Whitley, 2007) y han introducido nuevas formas de financiación (Braun, 1993; Heinze et al., 2009), instrumento clave para la orientación y promoción del sistema público de Investigación y Desarrollo (I+D).

Nuestro objeto de investigación son las intervenciones gubernamentales. En este artículo analizamos un caso: la política de financiación de la $\mathrm{I}+\mathrm{D}$ universitaria ${ }^{1}$ de un Gobierno regional (la Xunta de Galicia), en el periodo 2005-2018. Aunque el enfoque empleado es descriptivo, se pretende dar cuenta de algunos de los factores y procesos asociados al diseño de las políticas científicas, no solo desde una perspectiva política, sino también incluyendo los efectos de las instituciones y los instrumentos.

El marco analítico propuesto combina el enfoque de la economía política (Hall, 1993; Taylor, 2016), para comprender los resultados de los procesos de adopción de las políticas, con el análisis de políticas públicas desde el denominado policy design

1. Este análisis se circunscribe a los instrumentos de la Xunta impulsados desde la Consejería de Cultura, Educación y Universidad. No se han incluido otros instrumentos del Plan Gallego de I+D+i, que gestiona la Agencia Gallega de Innovación (GAIN), más relacionados con la innovación (González López 2019; González López y Guntín-Araujo, 2019; Vence, 2010). 
(Peters, 2018). Los Gobiernos desarrollan sus políticas por medio de instrumentos de naturaleza, funcionamiento y propósitos diversos (Hood y Margetts, 2007; Vedung, 1998). Así pues, suponemos que los procesos de introducción de instrumentos o las formas de asignación de recursos son factores relevantes en la compresión de cómo los Gobiernos seleccionan sus instrumentos (Capano y Lippi, 2017) y qué interacciones se producen en el contexto de los policy mix o carteras de instrumentos ${ }^{2}$. La investigación empírica sobre los instrumentos en este campo es limitada, pues este tipo de enfoque se ha introducido recientemente (Capano, 2018). Predominan aproximaciones desde el discurso (Elzinga y Jamison, 1995) o el análisis de programas específicos; por ejemplo, el Programa Ramón y Cajal (Cruz-Castro y Sanz-Menéndez, 2005). Por otro lado, la mayoría de estas investigaciones han adoptado un enfoque sincrónico o estático, y existen pocos análisis históricos de las políticas de fomento de la I+D; estudios de la secuencia, la evolución y el cambio de los instrumentos y de sus interacciones. Aquí adoptamos una aproximación causal de tipo histórico (Stinchcombe, 2005), que pretende comprender la construcción de las políticas a partir de su secuencia de desarrollo.

La nota de investigación se organiza del modo siguiente. La próxima sección esboza la pregunta de investigación subyacente y expone los principales factores explicativos considerados, así como los métodos y fuentes utilizados. La tercera sección argumenta la relevancia del caso gallego. La sección cuarta describe el marco institucional y de financiación universitaria. La quinta sección presenta los resultados más destacados del análisis y los relaciona con algunas conclusiones de estudios previos sobre políticas e instrumentos. Y, finalmente, la sección sexta formula algunas conclusiones, revisa las limitaciones y plantea líneas de investigación futuras.

\section{MARCO ANALÍTICO Y MÉTODOS}

La pregunta central de investigación quiere contribuir a entender la adopción de los instrumentos de política pública y analiza algunos de los factores o variables independientes e intervinientes que contribuyen a la explicación. El marco analítico adoptado asume gran parte del legado de la investigación previa: primero, la política y los procesos políticos, en el contexto de actuación de las Administraciones públicas, producen efectos en las modalidades, la orientación y la instrumentación de las políticas públicas. Estos efectos están mediados, entre otros factores, por las ideologías de los partidos en el Gobierno, la existencia o no de coaliciones, los intereses de los actores y la adopción de ideas, así como la emergencia de emprendedores de políticas públicas. Segundo, desde una perspectiva temporal, la arquitectura institucional existente — del Gobierno y de las Administraciones públicas—y la gobernanza multinivel

2. En este artículo los conceptos de instrumento y cartera de instrumentos (policy mix) se asocian con los términos administrativos al uso: programas, convocatoria, planes, etc. 
limitan el campo de las decisiones, crean restricciones en las conductas de los actores y definen la estructura de oportunidades. Tercero, los propios instrumentos de la intervención gubernamental se institucionalizan y se convierten en parámetros de la situación, sobre los cuales los actores sociales y políticos se alinean e intentan influir o modificar. La trayectoria dentro de la senda condiciona las respuestas adaptativas, aunque la emergencia de coyunturas críticas abre las puertas al cambio y a la generación de nuevas condiciones con efectos duraderos.

La aproximación causal adoptada en este trabajo no es determinística ni probabilística. Asumimos que la contribución a los efectos viene de fuentes diversas y sigue caminos variados; el efecto conjunto depende de las contribuciones de los orígenes separados, que pueden ser aditivos o contradictorios. En definitiva, suponemos que las "causas son condiciones INUS por sus efectos" (Mackie, 1965; Ragin, 2000), entendiendo que una condición INUS es una "parte Insuficiente pero Necesaria de una condición Innecesaria pero Suficiente para producir una contribución al efecto». Los estudios de causalidad histórica tienden a utilizar de modo predominante el análisis documental y las entrevistas con actores e informantes privilegiados.

Para la construcción de nuestra evidencia se ha utilizado una amplia variedad de materiales procedentes de distintas fuentes. Se han revisado las leyes de presupuestos de Galicia para analizar el volumen de fondos destinados a las universidades a lo largo del periodo 2005-2018. Se han clasificado las partidas presupuestarias según sus modalidades básicas: financiación generalista de las universidades (transferencias directas) o financiación de actividades específicas de $\mathrm{I}+\mathrm{D}$ (y sus convocatorias competitivas). Se han examinado todas las convocatorias públicas autonómicas de financiación de actividades de I+D en el Sistema Universitario de Galicia (SUG) —ayudas para: incorporación de recursos humanos, realización de proyectos de investigación, realización de actividades científicas de grupos de investigación, centros y unidades de I+D, etc.- con el objetivo de determinar la evolución de la cartera de instrumentos de financiación de la $\mathrm{I}+\mathrm{D}$ universitaria de la Consejería encargada de universidades (actualmente Consejería de Cultura, Educación y Universidad $^{3}$ ). Asimismo, se han identificado y examinado las normativas (europeas, estatales y autonómicas) que condicionan la puesta en marcha, funcionamiento e interacción entre instrumentos. De forma complementaria se han realizado entrevistas semiestructuradas en profundidad y se han mantenido reuniones con los gestores actuales de la Consejería en el curso del proyecto, así como con responsables de las universidades y con grupos de los centros y unidades de I+D del SUG. También se han realizado entrevistas informativas a responsables de la Consejería de Hacienda y a cargos de las universidades gallegas.

3. La Consejería encargada de universidades ha modificado su nombre en varias ocasiones a lo largo del periodo de análisis. Por cuestiones prácticas, a lo largo del artículo nos referimos a ella como Consejería de Universidades, aunque este no sea su nombre oficial. 


\section{EL CASO GALLEGO Y LAS CARTERAS DE INSTRUMENTOS DE FINANCIACIÓN DE LA INVESTIGACIÓN UNIVERSITARIA}

Dado que esta investigación sigue la estrategia del análisis de caso, es imprescindible explicitar de qué caso se trata (Gerring, 2016) la política de I+D universitaria de Galicia. La Xunta de Galicia, con Gobiernos conservadores, adoptó en los noventa un modelo singular de política de $\mathrm{I}+\mathrm{D}$ con atención a los intereses empresariales (Fernández et al., 2003; Sanz-Menéndez y Cruz-Castro, 2005). Posteriormente, la separación institucional de la política general de I+D de la de universidades, así como el desarrollo de una política de investigación para las universidades, hace de Galicia un caso desviado (Seawright y Gerring, 2008) y, por tanto, de valor exploratorio de nuevos factores causales y de contraste de las explicaciones determinísticas. Algunas características de la política de I+D de la Xunta hacen que también sea una oportunidad empírica para comprender los procesos de diseño y cambio de los instrumentos de la política pública ${ }^{4}$.

Galicia, como el resto de las CC.AA., recibió las transferencias y es responsable directa de la financiación de sus tres universidades públicas. Lo singular es que la Xunta, en una coyuntura crítica, desarrolló instrumentos de financiación de la I+D diferenciados de la financiación generalista para las universidades y separados de la política de investigación e innovación (Borrás y Jordana, 2016; González-López y Guntín-Araújo, 2019). Construyó, diseñó e implementó instrumentos diferenciados de fomento y promoción de las actividades de $\mathrm{I}+\mathrm{D}$ de las universidades, destinados a investigadores, grupos de investigación y unidades y centros de I+D. En otras CC. AA., en general, ha habido dos políticas: la de I+D (sin diferenciar los destinatarios) y la de universidades. Galicia es, pues, un caso singular de política de I+D universitaria de carácter dedicado, o de la evolución de las políticas universitarias en España, con líneas diferenciadas entre financiación basal (función docente para la organización en su conjunto) e investigación (a diversos actores en el seno de la universidad) que podría asemejarse a los modelos de financiación basados en resultados vigentes en otros países (Hicks, 2012).

Además, cuando se piensa en explicaciones generales que asocian la orientación política de los Gobiernos con la adopción de políticas e instrumentos de actuación (Cruz-Castro et al., 2004; Sanz-Menéndez y Cruz-Castro, 2005), el caso gallego también ofrece oportunidades. El sistema político gallego se ha caracterizado por una gran estabilidad. Desde 1990 ha habido Gobiernos mayoritarios del Partido Popular (PP), salvo entre 2005 y 2009, cuando hubo un Gabinete de coalición compuesto por el Partido dos Socialistas de Galicia-Partido Socialista Obrero Español (PSdeG-PSOE) y el Bloque Nacionalista Galego (BNG). Esta circunstancia permite analizar los efectos

4. A este respecto, se están realizando dos trabajos complementarios: uno que centra su atención en los efectos de los instrumentos en los centros de I+D y sus formas de autoridad y otro describiendo la cartera de instrumentos desarrollada por la Consejería de Universidades desde 2005. 
de los cambios de partidos en el Gobierno en las políticas implementadas, así como los efectos de los Gobiernos de coalición en el reparto competencial y en la dinámica de la política de $\mathrm{I}+\mathrm{D}$.

Asimismo, la Consejería de Universidades ha desarrollado instrumentos (regulatorios, financieros y discursivos) y una parte de la financiación destinada a las universidades ha pasado a focalizarse en los centros e institutos de investigación o en los grupos de investigación que trabajan en las universidades, lo que contribuye a un cambio en las estructuras de autoridad en la investigación (Cruz-Castro y Sanz-Menéndez, 2018) y aumenta la competición por los recursos. En resumen: competencias en materia de universidades, un modelo propio de financiación universitaria y el desarrollo de instrumentos específicos de política de $\mathrm{I}+\mathrm{D}$, que han ido cambiando y evolucionando a lo largo del tiempo, consolidan a Galicia como un caso de interés también para el análisis de las políticas de $\mathrm{I}+\mathrm{D}$ como mecanismo de transformación de las universidades.

\section{EL MARCO INSTITUCIONAL Y FINANCIERO DEL SISTEMA UNIVERSITARIO DE GALICIA}

Nuestro objeto de análisis son los instrumentos de la política de I+D, y el objetivo es comprender la continuidad y el cambio en las políticas; por ello, hay que situar su diseño, adopción y evolución en el contexto regulatorio, político y financiero apropiado. Para ello analizaremos, en primer lugar, la coyuntura crítica que se plasmó en el reparto competencial entre consejerías del Gobierno (y su persistencia posterior). Y, en segundo lugar, describiremos la evolución de la financiación de las universidades y la cartera de instrumentos de la I+D universitaria.

\section{Una politica de $I+D$ para las universidades}

Desde el punto de vista histórico hay dos hitos que han tenido consecuencias a largo plazo: el primero, el traspaso de competencias de la Administración General del Estado (AGE) a la Comunidad Autónoma y la creación de dos nuevas universidades, que se sumarían a la de Santiago de Compostela (Guntín Araujo y Fernández Prieto, 2015), pero este no es objeto de nuestra atención. El segundo, los efectos de los acuerdos de un Gobierno de coalición en Galicia (2005-2009) —el último Gobierno autonómico de coalición tras el tripartito encabezado por el socialista Fernando González Laxe (1987-1989) — en la distribución de las competencias de universidades e investigación e innovación en consejerías distintas. El Gobierno de coalición, sustentado por el PSdG-PSOE y el BNG, supuso un caso de coyuntura crítica (Collier y

5. La coyuntura crítica es una transición que configura la política futura, abriendo unas vías de acción política e imposibilitando otras. 
Collier, 1991) por la separación de políticas en distintas estructuras de la Administración pública autonómica, resultado del reparto competencial entre consejerías y sus consecuencias futuras.

Tras el nombramiento en 2005 de Emilio Pérez Touriño como presidente de la Xunta de Galicia ${ }^{6}$, la distribución de competencias en materia de universidades e investigación se modificó. Las consejerías de Educación y Ordenación Universitaria e Innovación e Industria ${ }^{7}$ pasaron a depender de cargos afines a dos partidos políticos distintos. La primera del PSdG-PSOE y la segunda del BNG. Posteriormente, la definición de las responsabilidades ${ }^{8}$ llevó a un cierto desdoblamiento de las competencias en $\mathrm{I}+\mathrm{D}$, creándose el germen de una diferenciación de la política en función de sus destinatarios. Hasta entonces, la Consejería de Universidades se encargaba fundamentalmente de la regulación, la financiación generalista de las universidades y los aspectos relativos a la enseñanza; mientras, Innovación e Industria era responsable de la financiación competitiva de las actividades de investigación, tanto de las universidades como de otras entidades, en el contexto del Plan Gallego de Investigación, Desarrollo e Innovación Tecnológica?.

Los nuevos responsables de la Consejería de Universidades, ante la ausencia de incentivos a las universidades para sus misiones investigadora y de transferencia, crearon una Dirección General de Promoción Científica y Tecnológica del SUG (DGPCyT-SUG), a la que se asignaron fondos ${ }^{10}$. La Consejería desarrolló acciones destinadas a la promoción de la investigación en las universidades, ello de forma complementaria con la financiación del Plan Gallego de I+D+i (PGIDIT). El Plan era gestionado por la Dirección General de Investigación, Desarrollo e Innovación, adscrita a la Consejería de Innovación e Industria, en manos del BNG. Así pues, la nueva DGPCyT-SUG comenzó a definir una política destinada a las universidades.

La victoria del Partido Popular en 2009 llevó a Alberto Núńez Feijóo a la Presidencia de la Xunta ${ }^{11}$. En contra de las expectativas habituales de cambio de orientación de las políticas públicas, se mantuvo la distribución competencial entre consejerías, aunque una se renombró como Economía e Industria ${ }^{12}$, que mantuvo la Dirección General de I+D+i, y se creó una Secretaría General de Universidades en la Consejería

6. Real Decreto 1008/2005, de 30 de julio (DOG de 1 agosto de 2005).

7. Decreto 211/2005, de 3 de agosto (DOG de 4 agosto de 2005).

8. Decreto 552/2005, de 20 de octubre (DOG de 8 noviembre de 2005) y Decreto 585/2005, de 29 de diciembre (DOG de 9 enero de 2006).

9. Este PGIDIT, aprobado en Consejo de Gobierno de la Xunta de Galicia en su reunión del 21 de septiembre de 2006, constituyó el marco de financiación autonómica de las actividades de investigación e innovación en el quinquenio 2006-2010.

10. Entrevista E4.

11. Real Decreto 630/2009, de 17 de abril (BOE de 18 abril de 2009).

12. Decreto 79/2009, de 19 de abril (DOG de 20 abril de 2009) y Decreto 83/2009, de 21 de abril (DOG de 22 abril de 2009). 
de Educación y Ordenación Universitaria ${ }^{13}$. Las actividades de I+D universitaria no solo continuaron siendo responsabilidad de la Consejería de Universidades, sino que su papel en la financiación de la I+D universitaria se fue reforzando. Dado que el foco tradicional de la política de I+D e innovación promovido por el PP gallego tenía énfasis empresarial (Fernández et al. 2003; Sanz-Menéndez y Cruz-Castro 2005), una explicación plausible es que el nuevo Gobierno encontró en el diseño de competencias existente una oportunidad para centrarse en sus propias prioridades, sin la interferencia de las universidades; además, estas se han ido mostrando críticas con el Gobierno. Así pues, la continuidad era conveniente y se evitaban potencialmente conflictos con sectores poco afines.

Esa coyuntura crítica ha dejado un legado institucional (Pierson, 2004; Rose, 1994) ${ }^{14}$ relevante en la configuración de la Administración pública autonómica, con efectos sobre las políticas e instrumentos. De hecho, este reparto competencial ha pervivido hasta ahora. Tras las elecciones autonómicas de julio 2020 y la nueva mayoría absoluta del PP, las competencias en innovación están en la Vicepresidencia Segunda y Consejería de Economía, Empresa e Innovación, mientras que la financiación de la $\mathrm{I}+\mathrm{D}$ universitaria ha permanecido, hasta el momento, en Universidades. Asimismo, hay que señalar otro factor: la importancia que tendrán las decisiones de emprendedores políticos (Schneider y Teske, 1992) ${ }^{15}$ procedentes de la universidad que se incorporan al Gobierno de coalición y a la Administración pública gallega. A estas variables del caso habría que agregar, adicionalmente, un marco general de acción política, complejo y fragmentado, derivado de los modelos actuales de gobernanza y de las características del sistema administrativo, altamente burocratizado y procedimental, que incentiva la economía de instrumentos.

\section{La financiación de la universidad y la cartera de instrumentos de la $I+D$}

Las misiones y actividades de las universidades gallegas se financian desde la Xunta por dos mecanismos complementarios, a los que se añade financiación finalista del Estado, Unión Europea (UE) y entidades privadas. Galicia cuenta con Planes de Financiación del SUG (PFSUG). Estos existen desde el 1990-96 (1996-99, 2000-03, 200510, 2011-15). El Plan de Financiación SUG actualmente en vigor (2016-2020) preveía destinar 2069,26 millones de euros a lo largo del periodo de cinco años (Xunta de Galicia, 2015). La financiación generalista ha mantenido a lo largo del tiempo una cierta

13. Decreto 324/2009, de 11 de junio (DOG de 17 junio 2009) y Decreto 332/2009, de 11 de junio (DOG de 24 junio 2009).

14. Las opciones políticas disponibles y las decisiones públicas se ven condicionadas por procedimientos institucionales y rutinas de los legados del pasado.

15. Este concepto se refiere a actores individuales con capacidad y posibilidad de influir de modo significativo en la dirección y flujo de las políticas realizadas. 
correspondencia con el número de estudiantes y el grado de experimentalidad de cada universidad (Fernández López y Vázquez García, 2013, 2017), por lo que puede decirse que ha seguido un patrón evolutivo de carácter incremental, como en la mayoría de los modelos autonómicos de financiación de las universidades (Larrán-Jorge y García-Correas, 2015; Pérez-Esparrells et al., 2018). Bien es verdad que desde 2013 se ha producido una creciente divergencia autonómica en las fuentes de financiación de las universidades, con muy variados niveles de contribución de las familias (subida de tasas), que confirman a Galicia como un caso singular de tasas académicas bajas (OSU, 2017).

Junto con los planes plurianuales de financiación, es necesario mencionar que en Galicia se ha ido dando un peso creciente de la financiación correspondiente a los programas competitivos de I+D universitaria, que ha pasado de representar el $3 \%$ al inicio del periodo hasta más del $15 \%$ del total de los recursos dirigidos al SUG en el 2018, como se observa en la figura 1. Esa proporción cada vez mayor de fondos competitivos en el total de recursos destinados a las universidades (fondos competitivos frente a financiación basal), muestra las dinámicas de transformación del sistema universitario, con una mayor capacidad de delimitación de los objetivos y mecanismos de seguimiento por parte del Gobierno.

\section{Figura 1.}

Evolución de la financiación universitaria y de la I+D por la Xunta de Galicia. Presupuesto total (escala de la derecha) y objetivos (en porcentaje del total)

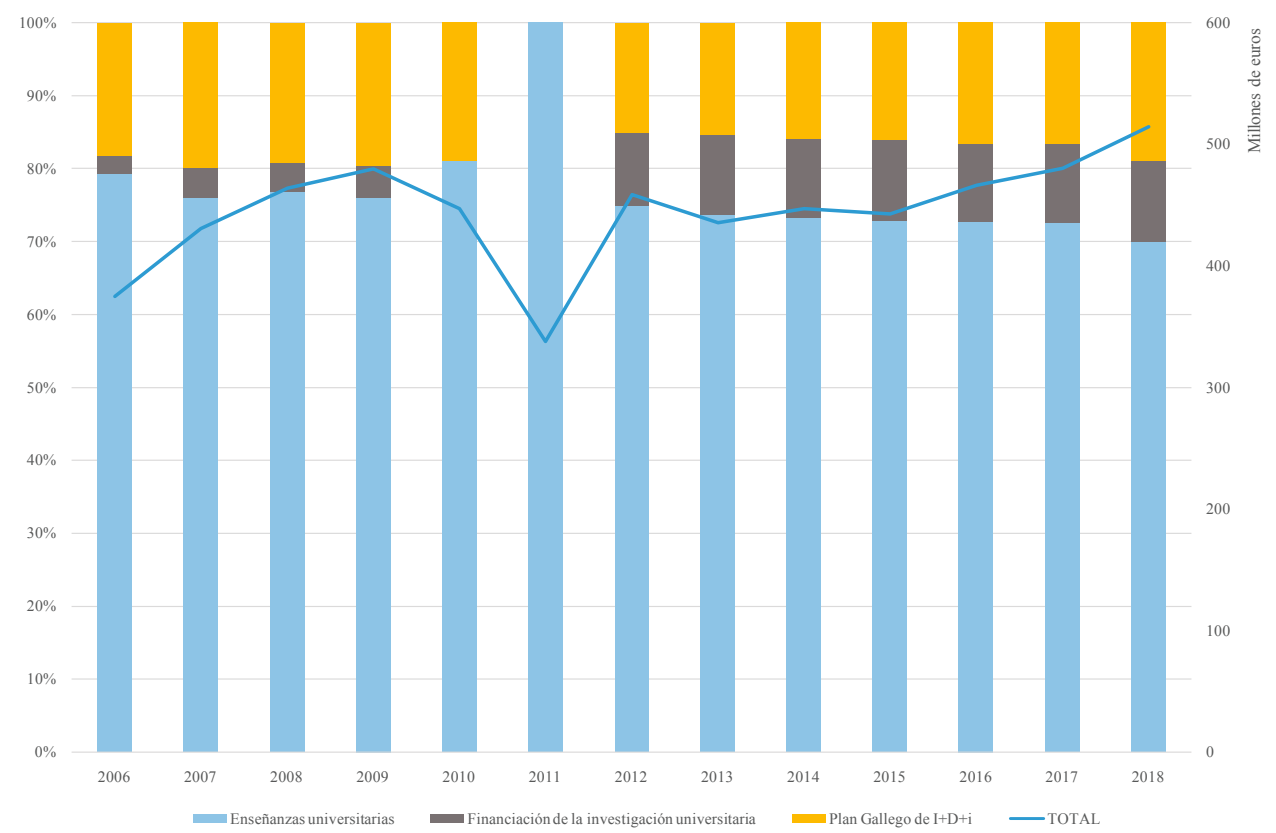

* Faltan los desgloses para los años 2010 y 2011.

Fuente: elaboración propia a partir de los datos de las leyes de presupuestos de la Xunta de Galicia. 


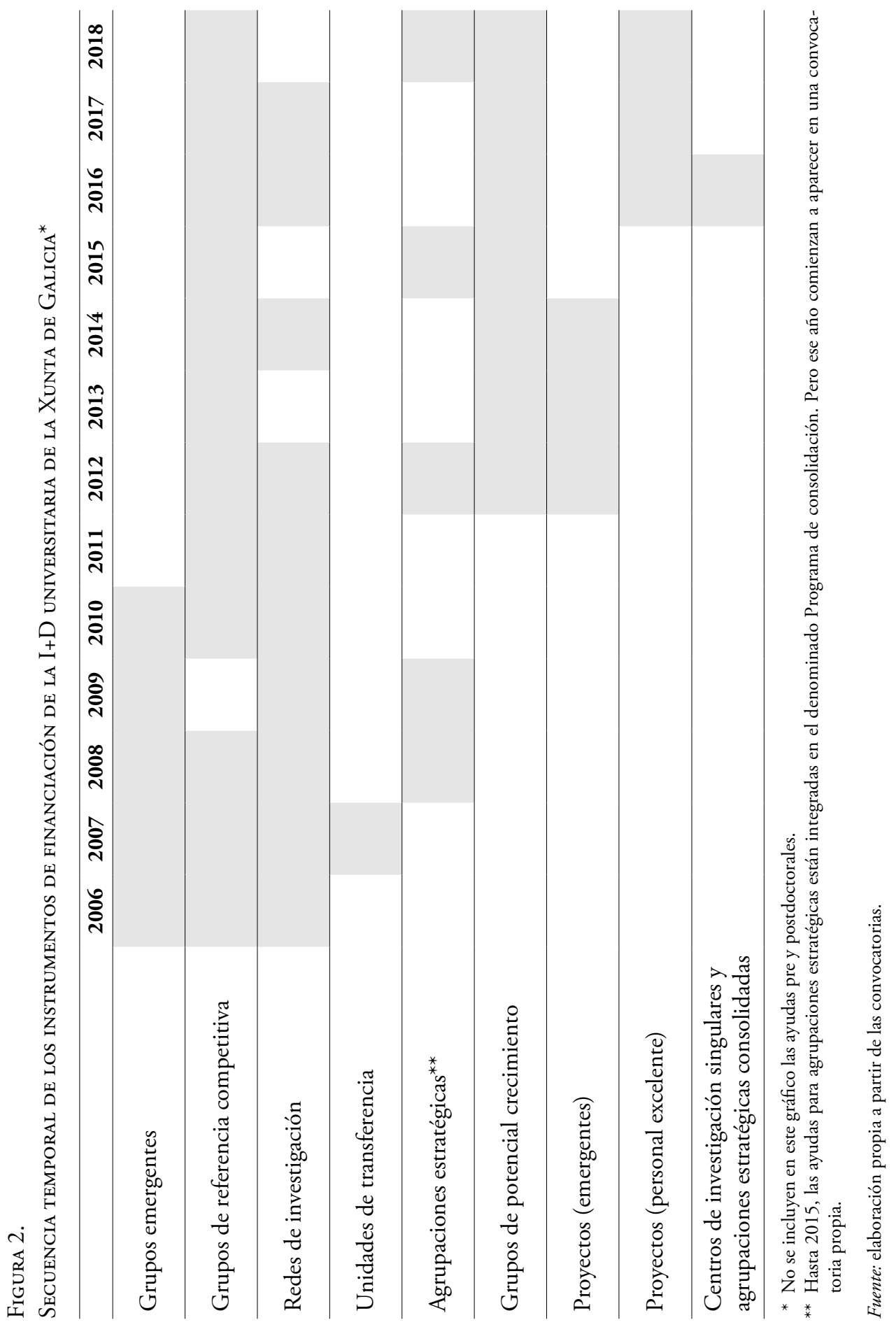


Entre 2006 y 2018 desde la Consejería de Universidades se han ido implementando (modificando y sustituyendo) hasta nueve tipos de ayudas competitivas destinadas a investigadores y, de manera destacada, a grupos de investigación y centros y unidades de $\mathrm{I}+\mathrm{D}$ (adicionalmente a las distintas ayudas de recursos humanos pre y postdoctorales que, con variaciones, ya existían previamente). Estas se presentan en la figura 2.

\section{DINÁMICAS EMERGENTES DE LAS POLÍTICAS Y LA CARTERA DE INSTRUMENTOS}

En esta sección se analizan algunas de las dinámicas emergentes en el diseño y adopción de las carteras de instrumentos para organizar las observaciones y resultados empíricos, a la luz de las variables independientes anteriormente expuestas.

\section{El diseño de los instrumentos y los condicionantes institucionales}

Los enfoques analíticos del estudio de las políticas suelen partir de dos posiciones diferenciadas con relación a la génesis de los instrumentos, su diseño y los condicionantes que influyen en su configuración: o bien las decisiones de los gestores públicos son racionales (incluso con racionalidad limitada) o bien estos están sometidos a múltiples restricciones institucionalizadas que se han interiorizado en su forma de actuar.

a. Actores movilizados en el sistema

Nuestro caso evidencia los límites de los enfoques racionales de adopción de los instrumentos. Los gestores públicos diagnostican, analizan, calculan, definen objetivos e incluso estiman los efectos y las consecuencias, pero lo hacen con restricciones. Los gestores están constreñidos por los actores movilizados del sistema (responsables de las universidades, investigadores, etc.) y sus preferencias, así como por las estructuras institucionales. Hemos comprobado que las decisiones tomadas sobre los instrumentos, sus características o los requisitos de los solicitantes, se ven influidas por interacciones de carácter formal e informal, entre los decisores y los académicos: reuniones de trabajo con vicerrectores y directores de centros de investigación, actos institucionales con presencia de miembros de la comunidad universitaria, etc. También constatamos que la cooperación con las partes interesadas se percibe como importante para la efectividad de las políticas. Por su parte, la pertenencia a los cuerpos de profesorado universitario de los cuatro consejeros de Universidades, así como la de muchos otros cargos con responsabilidades en materia de I+D, ponen de manifiesto la capacidad, reconocida en la literatura, de los emprendedores politicos universitarios para influir, configurar e incluso capturar las actividades que financian la investigación, aunque en el contexto de las reglas de operación de las Administraciones públicas. 
b. Legitimidad de la acción político-administrativa

En el análisis observamos que los gestores creen responder racionalmente a la identificación e interpretación de los problemas que se quieren resolver (fragmentación y déficit de masa crítica, entre otros). Y lo hacen de las siguientes formas: movilizando recursos —en el contexto de la competición política一, utilizando algunas de las soluciones o instrumentos disponibles, o buscando inspiración en otras Administraciones públicas. Pero también lo hacen de modo posibilista, identificando medidas que son aceptables para los actores implicados, gubernamentales y universitarios (singularmente: dotación de fondos adicionales a las universidades y fomento de estructuras de investigación), razonablemente coherentes con las estructuras y funcionamiento de las universidades y acordes a las preferencias de los actores. En ese sentido, centrar los esfuerzos en la utilización de instrumentos financieros (zanahorias), en lugar de en los regulatorios, es una estrategia que suele hacer ganar legitimidad frente a los beneficiarios. Ello porque este tipo de instrumentos permiten ajustes de la propia conducta de los actores universitarios, así como adaptaciones e interpretaciones de los objetivos de los instrumentos. Adicionalmente, se implica a los actores universitarios en los procesos de evaluación y selección competitiva de la financiación o se crean consejos asesores, con expertos de prestigio, que contribuyen a legitimar.

Ante un diagnóstico inicial (realizado en 2006) que detectó la fragmentación de las capacidades científicas e investigadoras del SUG, se buscaba cambiar la situación ${ }^{16}$. Los decisores propusieron soluciones y, a la vez, actuaban sobre uno de los desafíos esenciales del sistema universitario español: la ausencia de financiación basal de las actividades de $\mathrm{I}+\mathrm{D}$ en las universidades. La solución encontrada fue la puesta en marcha de instrumentos de financiación estructural y plurianual que incentivasen la consolidación de grupos de investigación que, si bien no facilita financiación a todos, lo hace por un mecanismo de evaluación por pares para el segmento más dinámico de la academia. Posteriormente, en 2008, se seguiría esta senda de actuación comenzando a financiar unidades y centros de $\mathrm{I}+\mathrm{D}$.

Aquí conviene destacar que la sustitución de los instrumentos existentes con anterioridad requirió, en la práctica, la emergencia de emprendedores políticos que, provenientes de la universidad, se hiciesen cargo de las políticas, en contextos de dinámicas de competición partidista (Gobierno de coalición con partidos distintos ocupando las carteras con competencias en I+D) como un factor subyacente de diferenciación. Se sabe de la importancia que tienen las decisiones de los emprendedores políticos,

16. Tal y como señala una de las personas entrevistadas, «en 2006 ya estaba gobernando el bipartito [...]. Se inicia un programa de financiación de los grupos de investigación; básicamente referida a los grupos de referencia competitiva [...]. Se observa que uno de los problemas que tiene el sistema gallego es la ausencia de masa crítica. Por eso se va a los grandes grupos, se potencia la generación de grandes grupos [...]; y, en un momento dado, estructuras más allá del grupo» (entrevista E4). 
especialmente en las coyunturas críticas. El subsistema (Sabatier, 1988) ${ }^{17}$ de políticas universitarias y de $\mathrm{I}+\mathrm{D}$ se caracteriza, en buena medida, por dos variables: la procedencia de los responsables políticos (en la mayoría de los casos, de la comunidad universitaria gallega) y la escasa distancia entre estos y los actores interesados/afectados por las políticas (profesores, cargos universitarios) ${ }^{18}$.

\section{c. Gobernanza multinivel}

Nuestro análisis constata los efectos de la complejidad de los entornos institucionales desde la perspectiva de la interacción entre niveles de gobierno. Por ejemplo, en un contexto de estabilización fiscal y presupuestaria de la Xunta ${ }^{19}$, los gestores públicos autonómicos han aprovechado la existencia de los fondos FEDER para mejorar la financiación de las universidades (por ejemplo, para la dotación de fondos a los centros y unidades de $\mathrm{I}+\mathrm{D}$ universitarias). La incorporación de algunas actuaciones de financiación de la I+D universitaria al Programa Operativo Galicia (2014-2020) ${ }^{20}$, en el contexto de la Estrategia de Especialización Inteligente (RIS3), evidencia la capacidad de los gestores no políticos de buscar y aprovechar las oportunidades disponibles.

Por otro lado, la interacción entre el Programa FEDER de la UE y el diseño de un instrumento autonómico resulta relevante por otro motivo. Pone de manifiesto la existencia de situaciones donde la condición de posibilidad para afrontar la solución a un problema identificado ha surgido en un proceso de política pública diferenciado. El uso de los fondos FEDER ilustra cómo, en determinados contextos, una solución

17. Aquí se entiende como «la interacción de actores de diferentes instituciones interesados en un área de política».

18. El presidente de la Xunta de Galicia (2005-2009), Emilio Pérez Touriño — profesor de Economía y antiguo vicerrector de Asuntos Económicos en la USC-, nombró como consejera a la profesora de Genética de la USC Laura Sánchez Piñón. Por su parte, el responsable de la nueva Dirección General encargada de la I+D universitaria fue Rogelio Conde-Pumpido, que estaba vinculado a distintos puestos de gestión universitaria también en la USC. Asimismo, el nuevo consejero de Innovación e Industria, Fernando Xavier Blanco Álvarez (político del BNG de Lugo y trabajador de Telefónica), nombró a Salustiano Mato, entonces vicerrector de Investigación de la Universidad de Vigo, como director general de I+D+i.

En el primer Gabinete encabezado por Alberto Núñez Feijóo, el responsable de Educación y Ordenación Universitaria fue Jesús Vázquez Abad (2009-2015), que provenía de la Universidad de Vigo, y había sido decano en la Facultad de Ciencias Empresariales y Turismo de Ourense. El patrón de nombrar como consejero a un profesor universitario se ha mantenido hasta la actualidad: Román Rodríguez González (aunque con experiencia previa en otros cargos políticos), titular de la USC (2015-2019), Carmen Pomar Tojo, también de la USC (2019-2020), y de nuevo Román Rodríguez González, en septiembre de 2020. Desde 2009 el secretario general de Universidades es José Alberto Díez de Castro, profesor de Economía de la USC.

19. La Ley $2 / 2011$, de 16 de junio, de disciplina presupuestaria y sostenibilidad financiera de Galicia (BOE, 20 de julio de 2011), establece políticas de gestión presupuestaria muy estrictas, sobre la idea del «principio de anualidad».

20. Disponible en: https://bit.ly/2UBG9dr. 
predefinida, con sus propios objetivos, puede ser utilizada e integrada en otros instrumentos. FEDER promueve la financiación de proyectos encaminados al desarrollo de las regiones y la corrección de desequilibrios. Basándose en ello, los gestores públicos regionales incluyeron líneas de actuación en el mismo que permitían la actuación en materia de $\mathrm{I}+\mathrm{D}$ y transferencia de tecnología; en un movimiento general en España y a escala europea que, en su forma más reciente, se plasmó en las Estrategias de Especialización Inteligente (Borrás y Jordana, 2016).

\section{Cambio en las políticas y evolución en los instrumentos}

La cartera de instrumentos existente nos indica la necesidad de estudiar las políticas en términos dinámicos. Por eso, este estudio reivindica un enfoque histórico que ayude a interpretar la evolución y el cambio de las políticas a través de sus instrumentos, y no solo como resultado de los cambios en los partidos de gobierno.

a. Coyunturas críticas y evolución de las carteras de instrumentos

La persistencia del reparto competencial en materia de I+D permite constatar los efectos estructurantes de las coyunturas críticas del pasado. Los diseños institucionales de la Administración autonómica gallega, resultado del Gobierno bipartito (2005-2009), se han reforzado tras la vuelta del PP al Gobierno (2009-actualidad), convirtiéndose la Consejería de Universidades en el principal financiador de la I+D universitaria. Como se ha señalado, el proceso de reparto de competencias entre partidos del Gabinete de coalición facilitó la innovación que comenzó a financiar actividades de I+D universitaria. El Gobierno monocolor asumió y reforzó la situación para reducir los costes derivados de una posible reforma de la estructura de competencias en $\mathrm{I}+\mathrm{D}$ y porque, además, era consistente con sus preferencias. Sin embargo, esta situación afecta al funcionamiento del sistema de investigación gallego, con las consiguientes dificultades potenciales para la integración de recursos y capacidades.

b. Dinámicas en la cartera de instrumentos

Aunque la arquitectura institucional fue estable tras el cambio del Gobierno, es necesario revisar los procesos de cambio que afectan a los instrumentos (Howlett, 2000; Peters, 2000), tales como la sustitución o la acumulación, y que responden a los cambios en el nivel del diseño de la política. El cambio político en Galicia, en un entorno de estabilidad de la Administración pública, contribuyó a crear las condiciones para avanzar en un proceso de desplazamiento de los instrumentos. El Gobierno de coalición promovió el paso, desde la tradicional financiación a través de proyectos de investigación y ayudas predoctorales y postdoctorales (recursos humanos), hacia una cartera de instrumentos más destinada a la financiación de las unidades y estructuras organizadas de la actividad científica (grupos de investigación y formación e integración de capacidades). Se modificaron los objetivos, 
los mecanismos y los destinatarios de los instrumentos. Adicionalmente, el cambio de objetivos y de orientación política de los decisores incluyó una idea, emergente entonces en algunas CC. AA.: evitar duplicar las actividades de fomento de la I+D del Estado, diferenciándose en los instrumentos.

No resulta sencillo evaluar esta transformación decisiva. Cabría plantear una serie de factores explicativos, que incluirían: a) la intención de los nuevos responsables de la Consejería de Universidades de diferenciar sus instrumentos de los de Industria, aunque guardando las formas (integración en el Plan Gallego de I+D+i); b) la búsqueda de transformaciones en el ecosistema universitario por parte de personas conscientes de su limitada permanencia en el tiempo en los puestos de responsabilidad; c) las diferencias de criterio entre consejerías dirigidas por personas afines a partidos políticos distintos, o d) la competencia entre responsables políticos provenientes de diferentes universidades del sistema.

Tras el cambio de Gobierno en 2009, con la orientación hacia las empresas de los responsables de la Consejería de Economía y la estabilidad de los equipos de funcionarios en la de Universidades, se mantuvo el enfoque instrumental, aunque quizás pueda verse también como un caso de «no decisión» en políticas públicas (Bachrach y Baratz, 1963). En los años siguientes (una vez adoptado el modelo por el nuevo Gobierno del PP y posteriormente estabilizado) se desarrolló la tendencia a la acumulación de instrumentos (Rayner y Howlett, 2009). Se ajustaron los instrumentos de apoyo a los grupos de investigación ${ }^{21}$ que, introducidos en 2006, continúan hasta la actualidad. Mientras, otros nuevos instrumentos se fueron agregando a lo largo de los años. Este es el caso de las ayudas para centros y unidades de I+D: creación de agrupaciones estratégicas (2008) o, posteriormente, de centros de investigación singulares y agrupaciones estratégicas consolidadas de Galicia (2016). El resultado de este proceso acumulativo es la coexistencia de un número elevado de instrumentos destinados a grupos objetivo diversos (investigadores, grupos de investigación y centros y unidades de $\mathrm{I}+\mathrm{D})$ que potencialmente generan desajustes.

En este contexto se puede observar el peso que tienen las generaciones anteriores de los instrumentos en la configuración de los nuevos. Se constata una cierta dependencia de la senda (Pierson, 2000), que bien pudiera asociarse a retornos crecientes de la adopción de modalidades relacionadas o, de otro modo, a la lógica que demandan los actores de medidas y actuaciones singulares para resolver problemas que surgen en la agenda. La política competitiva de financiación de la $\mathrm{I}+\mathrm{D}$ en las universidades gallegas parece confirmar la influencia de las decisiones pasadas en la formulación y diseño de nuevos instrumentos. Véase, por ejemplo, la modalidad de agrupaciones estratégicas consolidadas (2016) dentro del programa de centros, cuyo objetivo es mantener la financiación a las agrupaciones estratégicas financiadas en el pasado.

21. Las ayudas a "grupos de potencial crecimiento» (2012) pueden considerarse una continuación de las ayudas a "grupos emergentes» (2006). 


\section{CONCLUSIONES, LIMITACIONES Y PERSPECTIVAS DE INVESTIGACIÓN}

La configuración de las carteras de instrumentos despierta un gran interés en el campo de estudio de las políticas públicas. Este trabajo contribuye a la mejor comprensión del diseño, adopción y evolución de las carteras de instrumentos. El análisis del conjunto de instrumentos de financiación competitiva de la investigación científica universitaria de la Consejería Universidades de la Xunta de Galicia ofrece evidencia relevante de cara a una mejor comprensión de los diferentes factores que condicionan el diseño de instrumentos y la evolución de las carteras a lo largo del tiempo. El estudio de este caso ha tenido un carácter exploratorio, pero pueden extraerse algunas conclusiones.

Esta investigación, al tiempo que refuerza la evidencia sobre patrones de evolución de las carteras (acumulación de instrumentos, por ejemplo), pone de manifiesto la importancia de entender los factores desencadenantes de cambios en el conjunto de instrumentos. En este caso, el principal factor asociado al cambio en las políticas fue un cambio de Gobierno autonómico, una reestructuración de competencias en materia de I+D entre consejerías y el reemplazo de responsables políticos; no obstante, otros eventos pueden desempeñar potencialmente ese papel catalizador. Después, un nuevo cambio de Gobierno (que no implicó la sustitución de los funcionarios responsables) no supuso una modificación radical en el diseńo de los instrumentos adoptados anteriormente. Por otro lado, esta investigación ha destacado los efectos de la institucionalidad y de las decisiones pasadas sobre el diseńo de nuevos instrumentos. Parecen detectarse efectos estructurantes, derivados de coyunturas críticas, en los instrumentos cuando se institucionalizan, que condicionan el desarrollo futuro de las políticas públicas.

Este artículo destaca, a su vez, la búsqueda de instrumentalidad (eficacia) y legitimidad (aceptación de las políticas por los actores sociales) por parte de los políticos y gestores. No menos importante para comprender el diseño de las políticas es el rol de los emprendedores políticos que alcanzan puestos de responsabilidad en los Gobiernos. $\mathrm{Y}$, asimismo, el de actores académicos diversos que, sin formar parte de las estructuras gubernamentales, ejercen influencia sobre los responsables y funcionarios públicos. Finalmente, conviene recordar que es difícil hablar de carteras coherentes de instrumentos cuando los gestores públicos están, por un lado, fuertemente constreñidos por los actores en el sistema, la estructura competencial o la gobernanza multinivel y, por el otro, tan condicionados por su racionalidad limitada, información incompleta, atención focalizada y capacidades organizativas en Administraciones públicas generalmente infradotadas de medios.

La investigación presentada, como todo estudio de caso, tiene limitaciones. Necesita más información que permita conocer de forma robusta y fiable las dinámicas internas e interacciones entre los diversos actores y que han llevado a la situación actual en términos de estructura competencial de I+D y de diseño de los instrumentos concretos. Específicamente, a nivel de política, sería necesario, por ejemplo, obtener información directa que complementase los indicios disponibles sobre los determinantes que llevaron al Gobierno del PP en 2009 a mantener la estructura de competencias heredada. Por otro lado, en el plano del diseño de las políticas no resulta fácil 
conocer la composición y funcionamiento del subsistema de actores, especialmente en sus dimensiones informales, y su influencia en las decisiones.

Cara a futuras investigaciones sería necesario mejorar la comprensión sobre cómo los instrumentos interactúan entre sí, generando bien efectos positivos (sinergias), bien negativos (contradicciones y conflictos) en el sistema de I+D universitaria. También entender en qué medida, y a través de qué mecanismos, determinados actores en el sistema (profesores, autoridades universitarias, etc.) tienen capacidad para hacer valer sus preferencias en las decisiones gubernamentales y, en particular, en el diseño, implementación o terminación de los instrumentos, así como en su intensidad (por ejemplo, volumen relativo de fondos destinado al mismo con respecto al total de financiación disponible). No menos importante es obtener evidencia más robusta y avanzar en la sistematización del conocimiento, a través de estudios comparados, sobre cómo fenómenos políticos (en nuestro caso, repartos competenciales) condicionan el diseño e implementación de instrumentos $\mathrm{y}$, eventualmente, sus efectos en el sistema de $\mathrm{I}+\mathrm{D}$ universitaria.

\section{AGRADECIMIENTOS}

Este artículo es un resultado de investigación de los trabajos derivados de dos contratos de apoyo tecnológico entre la Secretaria General de Universidades de la Xunta de Galicia y el CSIC (Instituto de Políticas y Bienes Públicos). Luis Sanz Menéndez, desde 2018, es parte de la Comisión Asesora del programa de Centros de Investigación Singulares y Agrupaciones Estratégicas Consolidadas del Sistema Universitario de Galicia (DOG, 11 agosto de 2016). Los autores, habiendo recibido el CSIC financiación de la Xunta, manifiestan no tener conflicto de interés y señalan que sus análisis son independientes del financiador. Se agradecen las críticas y sugerencias recibidas de dos revisores anónimos y del editor de la RECP, así como los comentarios de Rogelio Conde, Faustino Infante y María Jesús Tallón a versiones anteriores de este trabajo. Los errores y déficits corresponden a los autores.

\section{Referencias}

Bachrach, Peter y Morton S. Baratz. 1963. «Decisions and Nondecisions: An Analytical Framework», The American Political Science Review, 57 (3): 632-642. Disponible en: https://doi.org/10.2307/1952568.

Borrás, Susana y Jacint Jordana. 2016. "When Regional Innovation Policies Meet Policy Rationales and Evidence: A Plea for Policy Analysis», European Planning Studies, 24 (12): 2133-2153. Disponible en: https://doi.org/10.1080/09654313.2 016.1236074.

Braun, Dietmar y Francoise-Xavier Merrien (eds.). 1999. Towards a New Model of Governance for Universities?: A Comparative View Higher Education Policy. Londres; Filadelfia: Jessica Kingsley Publishers. 
Braun, Dietmar. 1993. «Who governs intermediary agencies? Principal-agent relations in research policy-making», Journal of Public Policy, 13 (2): 135-162. Disponible en: https://www.jstor.org/stable/4007501.

Capano, Giliberto y Andrea Lippi. 2017. «How Policy Instruments Are Chosen: Patterns of Decision Makers' Choices», Policy Sciences, 50 (2): 269-293. Disponible en: https://doi.org/10.1007/s11077-016-9267-8.

Capano, Giliberto. 2018. «Policy Design Spaces in Reforming Governance in Higher Education: The Dynamics in Italy and the Netherlands", Higher Education, 75 (4): 675-94. Disponible en: https://doi.org/10.1007/s10734-017-0158-5.

Collier, Ruth Berins y David Collier. 1991. Shaping the Political Arena: Critical Junctures, the Labor Movement and Regime Dynamics in Latin America. Princeton, NJ: Princeton University Press.

Cruz-Castro, Laura y Luis Sanz-Menéndez. 2005. «Bringing Science and Technology Human Resources Back in: The Spanish Ramón y Cajal Programme», Science and Public Policy, 32 (1): 39-53. Disponible en: https://doi.org/ $10.3152 / 147154305781779687$.

Cruz-Castro, Laura y Luis Sanz-Menéndez. 2018. "Autonomy and Authority in Public Research Organisations: Structure and Funding Factors», Minerva, 56 (2): 135-60. Disponible en: https://doi.org/10.1007/s1 1024-018-9349-1.

Cruz-Castro, Laura, Luis Sanz-Menéndez y Marta Romero. 2004. «Convergencia y divergencia en las políticas deficiencia y tecnología de los gobiernos regionales", Revista Española de Ciencia Política, 11:31-70.

Elzinga, Aant y Andrew Jamison. 1995. «Changing Policy Agendas in Science and Technology», en Sheila M. Jasanoff, Gerald E. Markle, James C Peterson y Trevor J. Pinch (eds.), Handbook of Science and Technology Studies. Londres: SAGE Publications.

Fernández López, Sara y Alberto Vaquero García. 2013. «El modelo de financiación de la universidad gallega 2011-2015: previsión de fondos y balance», Revista Galega de Economía, 22 (1): 73-96. Disponible en: https://doi.org/10.15304/ rge.22.1.1270.

Fernández López, Sara y Alberto Vaquero García. 2017. «Luces y sombras del nuevo plan autonómico de financiación de las universidades gallegas (2016-2020)», Revista Galega de Economía, 26 (1): 89-104.

Fernández, María, Luis Sanz-Menéndez y Laura Cruz-Castro. 2003. «Diseño institucional y preferencias políticas: o cómo equilibrar los intereses académicos en la política de ciencia, tecnología e innovación gallega», Inguruak. Revista Vasca de Sociología y Ciencia Política, 35: 33-68.

Gerring, John. 2016. Case Study Research: Principles and Practices. Cambridge: Cambridge University Press. Disponible en: https://doi.org/10.1017/9781316848593.

González-López, Manuel y Xulia Guntín-Araujo. 2019. «Evolución da política galega de innovación: de cero á especialización intelixente», Revista Galega de Economía, 28 (2): 23-38. Disponible en: https://doi.org/10.15304/rge.28.2.6154.

González-López, Manuel. 2019. «Understanding Policy Learning in Regional Innovation Policies: Lessons from the Galician Case», Innovation: The European Journal 
of Social Science Research, 32 (1): 104-18. Disponible en: https://doi.org/10.1080 /13511610.2018.1519780.

Guntín-Araujo, Xulia y Lourenzo Fernández Prieto. 2015. «Universidades, sistema de innovación e políticas científicas e de investigación en Galicia. 25 anos da creación das novas universidades galegas", Sarmiento. Revista Galego-Portuguesa de Historia da Educación, 18-19: 7-21. Disponible en: https://doi.org/10.17979/srgphe.2015. 18-19.0.4039.

Hall, Peter A. 1993. «Policy Paradigms, Social Learning, and the State: The Case of Economic Policymaking in Britain", Comparative Politics, 25 (3): 275-96. Disponible en: https://doi.org/10.2307/422246.

Heinze, Thomas, Philip Shapira, Juan D. Rogers y Jacqueline M. Senker. 2009. "Organizational and institutional influences on creativity in scientific research", Research Policy, 38 (4): 610-623. Disponible en: https://doi.org/10.1016/j. respol.2009.01.014.

Hicks, Diana. 2012. «Performance-Based University Research Funding Systems», Research Policy, 41 (2): 251-61. Disponible en: https://doi.org/10.1016/j. respol.2011.09.007.

Hood, Christopher C. y Helen Z. Margetts. 2007. The tools of government in the digital age. Nueva York, NY: Macmillan International Higher Education.

Howlett, Michael. 2000. «Managing the «hollow state»: Procedural policy instruments and modern governance», Canadian Public Administration, 43 (4): 412-431. Disponible en: https://doi.org/10.1111/j.1754-7121.2000.tb01152.x.

Larrán-Jorge, Manuel y Ángel García-Correas. 2015. «influyen los modelos de financiación autonómicos en la eficiencia de las universidades públicas españolas?», Revista de Contabilidad, 18 (2): 162-173. Disponible en: https://doi.org/10.1016/j. rcsar.2014.06.001.

Mackie, John L., 1965. "Causes and Conditions», American Philosophical Quarterly, 12: 245-265. Disponible en: https://www.jstor.org/stable/20009173.

Observatori del Sistema Universitario (OSU). 2017. ¿Quién financia la universidad? Comparación entre comunidades autónomas en España, Europa y la OCDE, 2009-2015.

Pérez-Esparrells, Carmen, Eva de la Torre y José María Gómez-Sancho. 2018. «La relevancia de la financiación pública en las universidades españolas», Presupuesto y Gasto Público, 90: 169-190.

Peters, B. Guy. 2000. «Policy instruments and public management: bridging the gaps", Journal of Public Administration Research and Theory, 10 (1): 35-47. Disponible en: https://doi.org/10.1093/oxfordjournals.jpart.a024265.

Peters, B. Guy. 2018. Policy Problems and Policy Design. Northampton, MA: Edward Elgard. Disponible en: https://doi.org/10.4337/9781786431356.

Pierson, Paul. 2000. «Increasing returns, path dependence, and the study of politics», American Political Science Review, 94 (2): 251-267. Disponible en: https://doi. org/10.2307/2586011. 
Pierson, Paul. 2004. Politics in Time: History, Institutions, and social Analysis. Princeton: Princeton University Press.

Ragin, Charles C. 2000. Redesigning Social Research. Fuzzy-Set Social Science. Chicago: University of Chicago Press.

Rayner, Jeremy y Michael Howlett. 2009. "Introduction: Understanding integrated policy strategies and their evolution", Policy and Society, 28 (2): 99-109. Disponible en: https://doi.org/10.1016/j.polsoc.2009.05.001.

Rose, Richard. 1994. Inheritance in Public Policy: Change Without Choice in Britain. New Haven: Yale University Press.

Sabatier, Paul A. 1988. "An advocacy coalition framework of policy change and the role of policy-oriented learning therein", Policy Sciences, 21 (2-3): 129-168. Disponible en: https://doi.org/10.1007/BF00136406.

Sanz-Menéndez, Luis y Laura Cruz-Castro. 2005. «Explaining the Science and Technology Policies of Regional Governments», Regional Studies, 39 (7): 939-954. Disponible en: https://doi.org/10.1080/00343400500289945.

Schneider, Mark y Paul Teske. 1992. "Toward a theory of the political entrepreneur: evidence from local government", American Political Science Review, 86 (3): 737-747. Disponible en: https://doi.org/10.2307/1964135.

Seawright, Jason, y John Gerring. 2008. "Case Selection Techniques in Case Study Research: A Menu of Qualitative and Quantitative Options», Political Research Quarterly, 61 (2): 294-308. Disponible en: https://doi.org/10.1177/1065912907313077.

Stinchcombe, Arthur L. 2005. The Logic of Social Research. Chicago: The University of Chicago Press.

Taylor, Mark Z. 2016. The Politics of Innovation: Why Some Countries Are Better Than Others at Science and Technology. Nueva York: Oxford University Press. Disponible en: https://doi.org/10.1093/acprof:oso/9780190464127.001.0001.

Vedung, Evert. 1998. "Policy Instruments: Typologies and Theories», en MarieLouise Bemelmans-Videc, Ray C. Rist y Evert Vedung (eds.), Carrots, Sticks and Sermons: Policy Instruments and Their Evaluation. New Brunswick, NJ: Transaction Publishers.

Vence Deza, Xavier. 2010. «Sistema gallego de innovación y políticas de innovación: balance de una década», Revista Galega de Economía, 19, 1-30.

Whitley, Richard. 2007. "The changing governance of the sciences", en Richard Whitley y Jochen Gläser (eds.), The Changing Governance of the Sciences: The Advent of Research Evaluation Systems. Dordrecht: Springer. Disponible en: https:// doi.org/10.1007/978-1-4020-6746-4.

Whitley, Richard. 2011. "Changing governance and authority relations in the public sciences», Minerva, 49 (4): 359-385. Disponible en: https://doi.org/10.1007/ s11024-011-9182-2.

Presentado para evaluación: 18 de junio de 2020.

Aceptado para publicación: 5 de noviembre de 2020 . 
MANUEL PEREIRA-PUGA

Manuel.Pereira@csic.es

Doctor en Sociología por la Universidad de A Coruña, gracias a una beca del Programa Nacional de Formación de Profesorado Universitario (FPU). Actualmente trabaja en el Instituto de Políticas y Bienes Públicos (IPP) del CSIC e imparte docencia en la Universidad Carlos III de Madrid. Manuel Pereira-Puga investiga sobre sistemas y políticas públicas de educación superior universitaria y de I+D, así como sobre la intersección entre ciencia, tecnología y sociedad desde una perspectiva interdisciplinar.

\section{LUIS SANZ-MENÉNDEZ}

Luis.Sanz@csic.es

Profesor de investigación del CSIC en el Instituto de Políticas y Bienes Públicos (IPP). Doctor en Sociología y Ciencias Políticas por la Universidad Complutense de Madrid (UCM), ha publicado más de un centenar de trabajos sobre los sistemas y políticas públicas de ciencia, tecnología e innovación. Sus trabajos han aparecido en revistas como: Research Policy, Minerva, Research Evaluation, Science and Public Policy, Technological Forecasting and Social Change, Scientometrics, Regional Studies, Public Understanding of Science o PLOS One. También ha escrito varios libros en estos campos. 